\title{
The vestigial enzyme D-dopachrome tautomerase protects the heart against ischemic injury
}

\author{
Dake Qi, ${ }^{1,2}$ Kwame Atsina, ${ }^{1,2}$ Lintao Qu, ${ }^{3}$ Xiaoyue Hu, ${ }^{1,2}$ Xiaohong Wu, ${ }^{1,2}$ Bin Xu, ${ }^{1,2}$ Marta Piecychna, ${ }^{4}$ Lin Leng, ${ }^{4}$ \\ Günter Fingerle-Rowson, ${ }^{5}$ Jiasheng Zhang, ${ }^{1,2}$ Richard Bucala, ${ }^{4}$ and Lawrence H. Young ${ }^{1,2,6}$
}

'Section of Cardiovascular Medicine, Department of Internal Medicine, ${ }^{2}$ Yale Cardiovascular Research Center, ${ }^{3}$ Department of Anesthesia, and ${ }^{4}$ Section of Rheumatology, Department of Internal Medicine, Yale University School of Medicine, New Haven, Connecticut, USA. ${ }^{5}$ Clinic I for Internal Medicine, Hematology and Oncology, University Hospital Cologne, Cologne, Germany. ${ }^{6}$ Department of Cellular and Molecular Physiology, Yale University School of Medicine, New Haven, Connecticut, USA.

\begin{abstract}
The cellular response to stress involves the recruitment and coordination of molecular signaling pathways that prevent cell death. D-dopachrome tautomerase (DDT) is an enzyme that lacks physiologic substrates in mammalian cells, but shares partial sequence and structural homology with macrophage migration inhibitory factor (MIF). Here, we observed that DDT is highly expressed in murine cardiomyocytes and secreted by the heart after ischemic stress. Antibody-dependent neutralization of secreted DDT exacerbated both ischemia-induced cardiac contractile dysfunction and necrosis. We generated cardiomyocyte-specific DDT knockout mice (Myh6-Cre $D d t^{f / f}$ ), which demonstrated normal baseline cardiac size and function, but had an impaired physiologic response to ischemia-reperfusion. Hearts from Myh6-Cre Ddt ${ }^{f / f l}$ mice exhibited more necrosis and LV contractile dysfunction than control hearts after coronary artery ligation and reperfusion. Furthermore, treatment with DDT protected isolated hearts against injury and contractile dysfunction after ischemia-reperfusion. The protective effect of DDT required activation of the metabolic stress enzyme AMP-activated protein kinase (AMPK), which was mediated by a CD74/CaMKK2-dependent mechanism. Together, our data indicate that cardiomyocyte secretion of DDT has important autocrine/paracrine effects during ischemia-reperfusion that protect the heart against injury.
\end{abstract}

\section{Introduction}

Myocardial ischemia resulting from coronary arterial occlusion leads to myocardial necrosis, arrhythmias, contractile dysfunction, and heart failure. Cellular adaptation to ischemic stress requires the coordination of cellular metabolic pathways to maintain energy homeostasis (1). AMP-activated protein kinase (AMPK) is a serine-threonine kinase that serves as a fuel gauge and coordinates energy-generating glucose uptake $(2,3)$ and glycolysis (4), while downregulating the energy-consuming synthesis of proteins (5) and lipids (6). AMPK also induces autophagy (7), prevents ROS production (8), and inhibits mitochondrial transition pore opening (9) during ischemia-reperfusion. Thus, the endogenous AMPK pathway preserves cellular ATP content, limits apoptosis and necrosis, and improves functional recovery of the heart during ischemia-reperfusion $(2,10)$. In addition, AMPK is emerging as a potential target for therapy in diabetes and ischemic heart disease. Although AMPK is primarily activated by cellular energy depletion, it is also regulated by hormones such as leptin and adiponectin that modulate metabolism $(11,12)$.

In the heart, there is emerging evidence that autocrine/paracrine factors also modulate cardiac metabolism, cell growth, and response to injury (13-15). We previously demonstrated that mac-

Conflict of interest: Yale University has applied for a patent describing the use of DDT in protecting against ischemic injury; Richard Bucala and Lawrence $\mathrm{H}$. Young are coinventors on this application. Unrelated to this research, Lawrence $\mathrm{H}$. Young is the recipient of a research grant from Merck Pharmaceuticals.

Submitted: September 4, 2013; Accepted: May 15, 2014

Reference information: / Clin Invest. 2014;124(8):3540-3550. doi:10.1172/JCI73061. rophage migration inhibitory factor (MIF) is highly expressed in cardiomyocytes and regulates cellular metabolism (15). MIF is better known as a prototype cytokine that regulates macrophage function in inflammation and plays an important role in innate immunity (16). However, endogenous MIF protects the heart by stimulating AMPK activation during experimental ischemiareperfusion and limiting cardiac apoptosis and necrosis $(15,17)$. Furthermore, functional polymorphisms in the human MIF gene promoter, which lead to reduced MIF expression and secretion, interfere with MIF's regulation of critical cellular signaling pathways $(15,18)$. However, MIF treatment also reduces cardiac contractility (19) and might increase inflammation after severe ischemic injury (17), which limits its potential development as a clinical treatment of ischemic heart disease.

D-dopachrome tautomerase (DDT) is an enzyme that catalyzes the tautomerization and decarboxylation of the non-naturally occurring substrate D-dopachrome to 5,6-dihydroxyindole (20, 21). DDT may trace its origin to the invertebrate melanotic encapsulation response, which is a primitive immune defense against tissue invasion (16). However, DDT is present in many mammalian tissues and is highly conserved among species (16). DDT has limited structural homology with the cytokine MIF; the DDT and MIF amino acid sequences share 34\% homology in humans and $27 \%$ in mice (22). Similar to MIF, the human DDT protein folds to form a homotrimer and is a ligand for the MIF receptor CD74 in macrophages (22). However, the physiologic action of DDT in the heart is currently unknown. Thus, we tested the hypothesis that DDT acts as an endogenous metabolic stress protein in the heart 
and evaluated whether the DDT pathway might have therapeutic potential for preventing cardiac injury during ischemia.

\section{Results}

Heart-derived DDT has protective autocrine/paracrine effects during ischemia-reperfusion. We found for the first time that DDT was highly expressed in cardiomyocytes, based on immunohistochemistry staining of mouse heart sections (Figure 1A). Brief ischemic stress triggered the secretion of DDT from preformed stores into the extracellular space in ex vivo isolated perfused mouse hearts, resulting in a 2 -fold increased DDT concentration in the coronary venous effluent (Figure 1B). Depletion of cardiomyocyte DDT was also readily apparent after in vivo ischemiareperfusion (Figure 1, A and $\mathrm{C}$ ).

In order to determine the physiologic effect of endogenously secreted DDT on ischemic tolerance, ex vivo isolated mouse hearts were perfused with or without anti-DDT neutralizing antibody and then subjected to ischemia-reperfusion. This antibody has high specificity for DDT and does not have immunoreactivity to MIF (22). The product of LV developed pressure and heart rate (referred to herein as the LVDP.HR index; used as an indicator of cardiac contractile function) recovered to only $30 \%$ of baseline after ischemia-reperfusion in hearts treated with anti-DDT, compared with $70 \%$ of baseline with nonimmune IgG treatment (Figure 1D). Similar differences were seen for other indices of cardiac contractile function, including LV developed pressure (LVDP), $+\mathrm{LV} d P / d t$, and $-\mathrm{LV} d P / d t$ (Supplemental Figure 1; supplemental material available online with this article; doi:10.1172/ JCI73061DS1). The extent of necrosis, assessed by histologic analysis after 2, 3, 5-triphenyltetrazolium chloride (TTC) vital staining, was also 2-fold greater in hearts perfused with anti-DDT (Figure 1E). Together, these results indicate that DDT is a secreted protein that functions via an autocrine/paracrine mechanism to prevent injury during ischemia.

The AMPK pathway is an essential component of the cellular stress response and defends the heart against ischemia $(1,2,10)$. In order to determine whether AMPK contributes to the cardioprotective effect of intrinsic DDT during ischemia, we assessed AMPK pathway activation by immunoblotting for phosphorylation of AMPK (at the Thr ${ }^{172}$ activating site in the catalytic domain) and its downstream target protein, acetyl-CoA carboxylase (ACC) (1). Ischemia-induced AMPK pathway activation was blunted by $43 \%$ in hearts treated with anti-DDT antibody (Figure 1F), which indicates that endogenously secreted DDT modulates cardiomyocyte metabolic stress signaling during ischemia.

Neutralization of endogenously secreted DDT inhibits hypoxiainduced AMPK activation in cardiomyocytes. Although cardiomyocytes constitute the predominant mass of the heart, there are an equal number of other cells, including fibroblasts, endothelial cells, and smooth muscle cells, that might also contribute to DDT's actions during ischemia. To test whether cardiomyocytes per se have an operative DDT autocrine pathway, we subjected isolated adult ventricular cardiomyocytes to hypoxia with and without anti-DDT. Hypoxic activation of the AMPK signaling pathway was diminished by $40 \%$ in the presence of anti-DDT antibody (Supplemental Figure 2), which indicates that cardiomyocytes secrete and respond to DDT.
Hearts with cardiomyocyte-specific DDT deletion have normal baseline structure and function, but impaired stress response to ischemia. To assess the physiologic role of cardiomyocyte-derived DDT in the intact heart, we generated cardiomyocyte-specific DDT knockout mice (Myh6-Cre Ddt t/fl; Figure 2A). These mice demonstrated a $90 \%$ overall reduction in DDT expression in heart homogenates compared with Ddt/flt and Myh6-Cre Ddt $t^{+/+}$controls, with residual DDT expression confined to large blood vessels after immunohistochemical staining of LV myocardium with anti-DDT specific antibody (Figure 2B). Myh6-Cre Ddt/f/l mice demonstrated normal baseline cardiac morphology and histology (Supplemental Figure 3) as well as intact LV size, wall thickness, and ejection fraction by echocardiography (Figure 2C), and their hearts had normal LV contractile function ex vivo (Figure 2D).

In order to examine whether cardiomyocyte DDT is an important component of the stress response in the heart, we assessed whether DDT deletion exacerbates the physiologic response to ischemia-reperfusion. We compared Myh6-Cre Ddt t/f/ hearts with both $\mathrm{Myh6}$-Cre $\mathrm{Ddt} t^{+/+}$and $\mathrm{D} d t^{t / f t}$ hearts as controls. Deletion of cardiomyocyte DDT exacerbated LV contractile dysfunction, with less recovery of the LVDP·HR index as well as other parameters of $\mathrm{LV}$ contractile function, in ex vivo perfused hearts after ischemia-reperfusion (Figure 2D and Supplemental Figure 4). Myh6-Cre Ddt $t^{f / f l}$ hearts also had 2.3-fold more histological necrosis compared with control hearts, based on the results of TTC vital staining (Figure 2, E-I).

To determine whether the susceptibility of Myh6-Cre Ddt/f/l hearts was due to the acute or chronic lack of cardiomyocyte DDT, these hearts were also perfused with buffer containing a physiologic concentration of recombinant DDT (rDDT; $50 \mathrm{ng} / \mathrm{ml}$ ). The recovery of $\mathrm{LV}$ contractile function and the extent of injury after ischemia-reperfusion was similar in Myh6-Cre Ddt $t^{f / f}$ hearts perfused with rDDT and control hearts (Figure 2, D-I). These results indicate that acute treatment with extracellular DDT is sufficient to rescue the phenotype and provide evidence that the predominant abnormality in the Myh6-Cre Ddt/f/f hearts is their lack of DDT.

To assess whether Myh6-Cre Ddt/f/f mice also had greater susceptibility to cardiac injury in vivo, they and Myh6-Cre $\mathrm{Ddt} t^{+/+}$and $D d t^{f / f l}$ control mice were subjected to transient left coronary artery ligation for 20 minutes followed by 3 hours of reperfusion. The resulting regional ischemia produced more severe cardiac injury in Myh6-Cre Ddt $t^{f / f}$ versus control mice, as evidenced by 3.5-fold greater necrosis on TTC staining of LV sections and 2.5-fold higher serum troponin release (Figure 3, A and B). Myh6-Cre Ddt/f/f mice also demonstrated impaired cell signaling with attenuated AMPK pathway activation during ischemia compared with both control groups (Figure 3C). In order to determine whether increased necrosis in Myh6-Cre $D d t^{A / f}$ mice resulted in greater contractile dysfunction 24 hours after ischemia-reperfusion, we assessed regional contractility in vivo by echocardiography. LV fractional shortening was 50\% lower in Myh6-Cre Ddt//ft versus Ddt/f/t control mice after ischemia-reperfusion (Figure 3D). Taken together, these results indicate that cardiomyocyte DDT has an intrinsic protective action to limit myocardial injury and resultant cardiac dysfunction after ischemia-reperfusion in the mouse heart.

DDT activates AMPK through a CaMKK-dependent mechanism. To understand the molecular mechanisms that transduce the ac- 
A
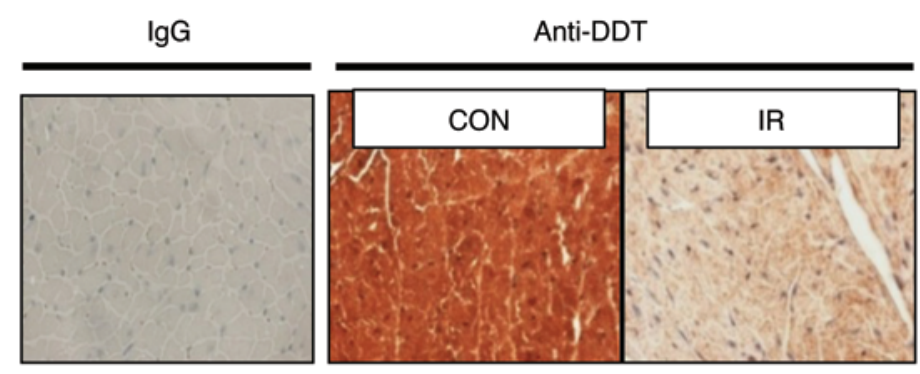

D

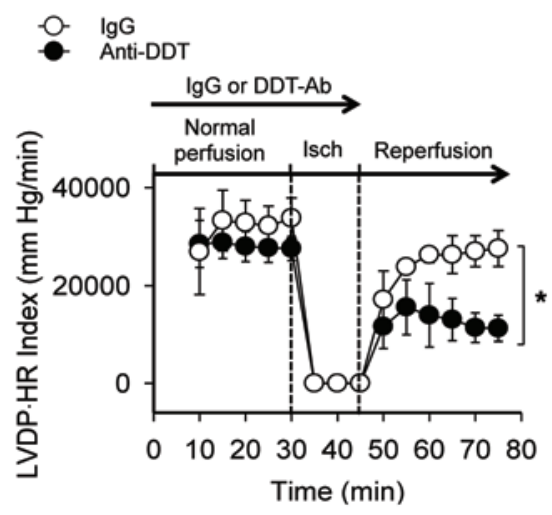

B

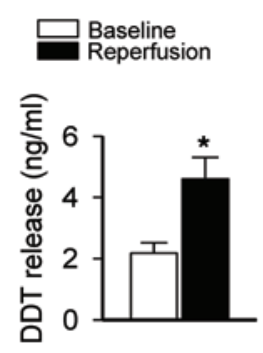

c

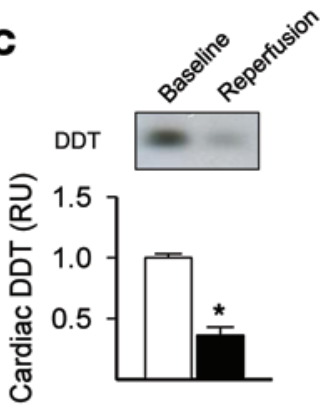

E

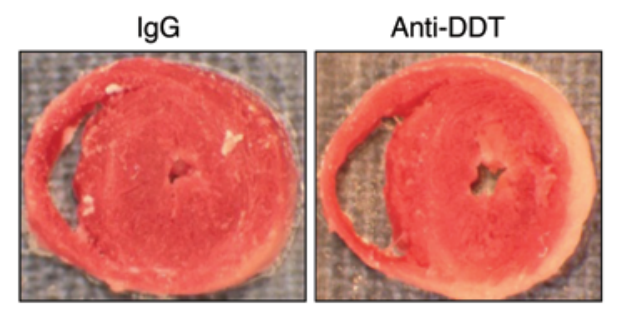

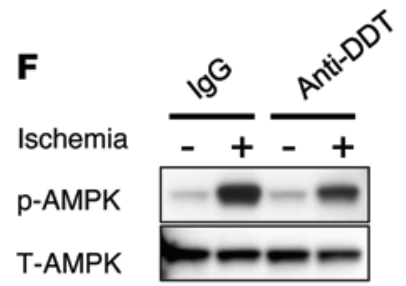

T-AMPK

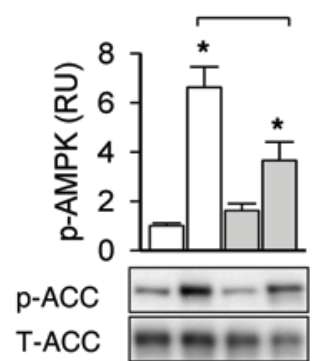

Figure 1. Heart-derived DDT has protective autocrine/paracrine effects during ischemia-reperfusion. (A) DDT expression was determined in mouse heart using immunohistochemistry with a polyclonal anti-DDT antibody or nonimmune IgG antibody (as a control for nonspecific staining). Heart LV sections were studied after 20 minutes of ischemia and 3 hours of reperfusion (IR) induced by coronary artery ligation or control sham surgery (CON). Original magnification, $\times 10$. (B) Cardiac DDT release into the coronary venous effluent was quantified by ELISA in ex vivo perfused hearts, during either normal baseline perfusion or global ischemia (15 minutes) and 30 minutes of reperfusion. (C) DDT immunoblots of heart homogenates from mice subjected to 20 minutes of ischemia and 3 hours of reperfusion or sham surgery (Baseline). (D) Cardiac contractile function (LVDP•HR index) in isolated mouse hearts treated with anti-DDT or nonimmune control IgG prior to 15 minutes of ischemia and 30 minutes of reperfusion. (E) Myocardial necrosis, determined by vital staining with TTC and expressed as the infarct area as a percentage of ventricular tissue in perfused hearts subjected to ischemia-reperfusion as in B. (F) Phosphorylation of AMPK and downstream ACC, detected on immunoblots of homogenates from perfused mouse hearts treated with anti-DDT or nonimmune IgC prior to 15 minutes of ischemia. Data are mean \pm SEM, $n=4-6$ per group. ${ }^{*} P<0.05$ vs. respective control or as indicated by brackets.

tions of DDT, we expressed and purified mouse rDDT, as previously described (22). Initial studies in ex vivo isolated rat heart muscles showed that rDDT stimulated AMPK and downstream ACC phosphorylation 2- to 3-fold, in a rapid and dose-dependent manner (Supplemental Figure 5, A and B). rDDT treatment also stimulated ${ }^{3} \mathrm{H}$-2-deoxyglucose uptake 2 -fold in these heart muscles (Supplemental Figure 5C), indicating stimulation of the initial step in the glucose metabolic pathway. In order to verify a specific cardiomyocyte action, we also treated isolated adult cardiomyocytes with rDDT and observed enhanced AMPK activation as early as 5 minutes, with peak activity at 15 minutes (Figure 4 A).

The mechanism of activation of AMPK by cytokines and hormones is not well understood (1). AMPK activation is primarily mediated by phosphorylation of the $\mathrm{Thr}^{172}$ activating site in its $\alpha$ catalytic subunit by upstream kinases, including liver kinase B (LKB1) and $\mathrm{Ca}^{2+} /$ calmodulin-activated kinase kinase (CaMKK) (1, 23). In order to test whether CaMKK might be primarily responsible, we initially treated cardiomyocytes with the CaMKK inhibitor STO-609 and found that rDDT activation of AMPK was prevented
(Figure 4B). Of the 2 CaMKK isoforms, CaMKK2 (also known as CaMKK $\beta$ ) has greater activity as an AMPK kinase than CaMKK1 (also known as CaMKK $\alpha$ ) $(23,24)$, but the heart also expresses CaMKK1, and both isoforms are inhibited by STO-609 (25). In addition, STO-609 has potential nonspecific pharmacologic effects that inhibit kinases other than CaMKK, including ERK8, Src, and CK2 (26). Thus, we used a genetic approach and directly assessed rDDT activation of the AMPK pathway in isolated perfused hearts from Camkk2 $2^{--}$mice (27). rDDT stimulated AMPK signaling in a dose-dependent manner in WT hearts, but Camkk2 deletion prevented activation of AMPK (Figure 4, E and F), indicative of a critical role for this isoform in transducing the action of DDT.

We then assessed whether DDT might initiate CaMKK signaling via an increase in intracellular calcium, by measuring diastolic calcium with fura-2 fluorescence in quiescent adult rat cardiomyocytes. rDDT treatment increased intracellular calcium concentration after 5 minutes of exposure, with return to baseline after 2 minutes (Figure 4C and Supplemental Figure 6). We further assessed the potential role for calcium signaling in DDT 
A

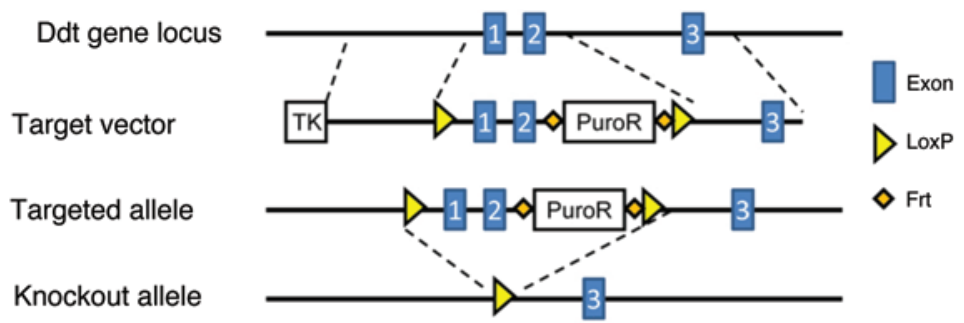

B

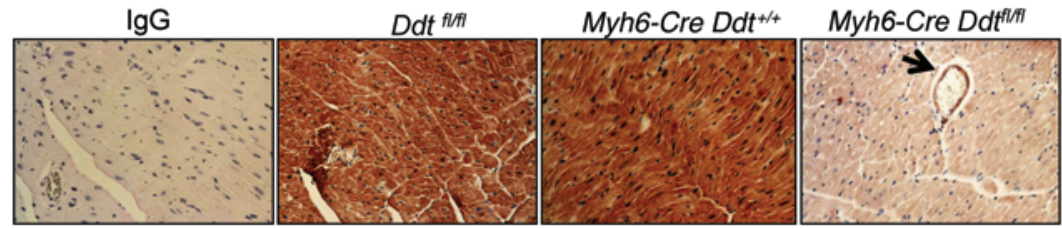

C
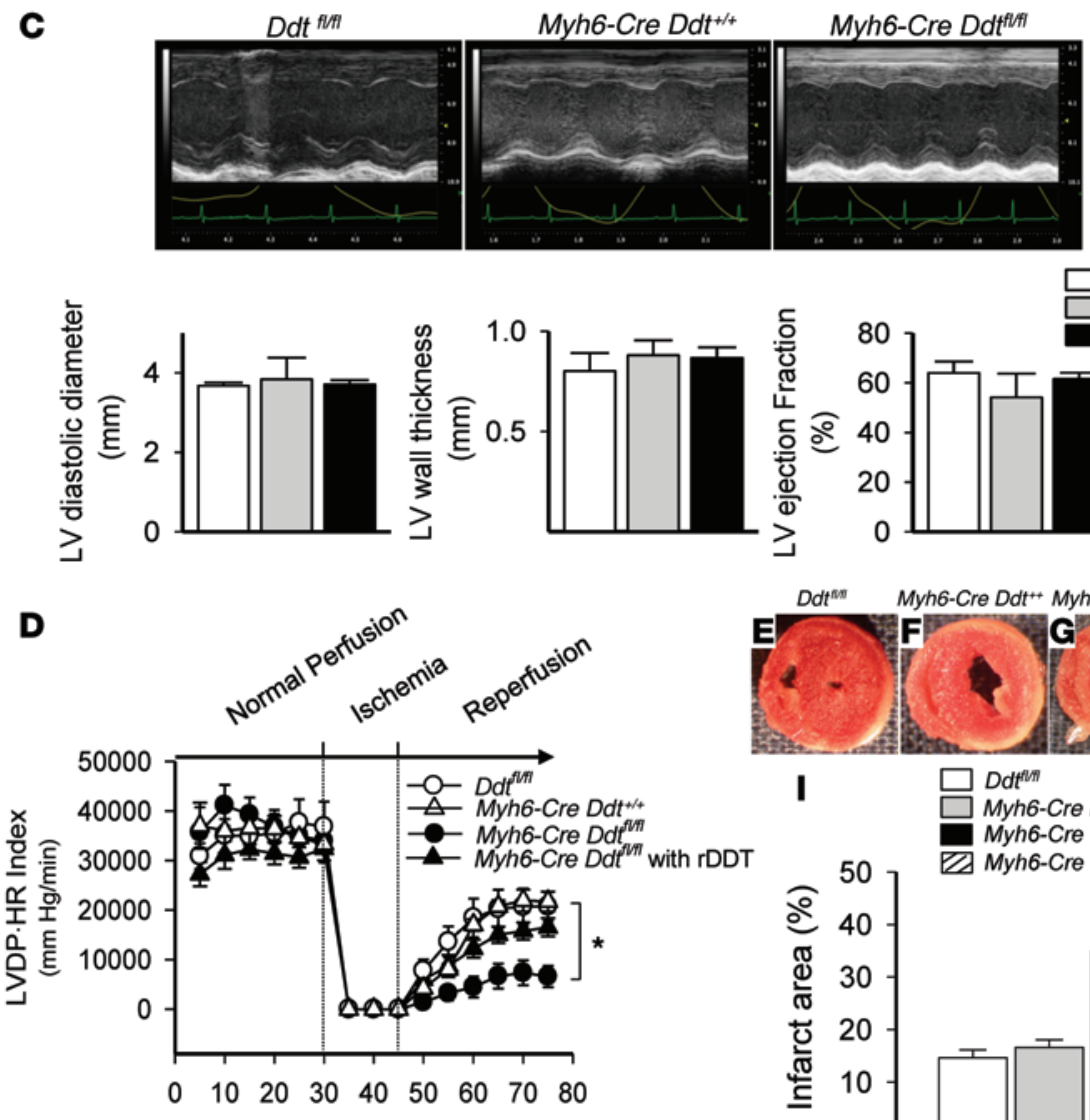

$D d t^{t}$ IT

Myh6-Cre Ddtt+t Myh6-Cre Ddtw

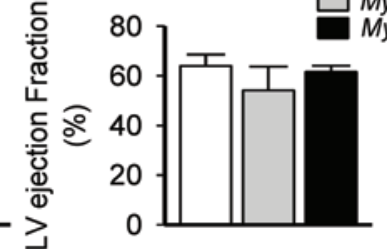

$D d t^{n w}$

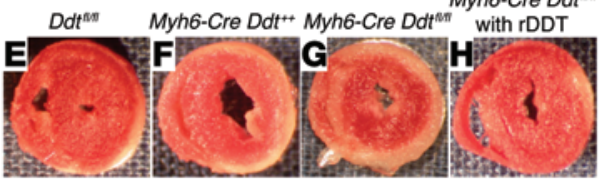

I

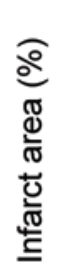

Figure 2. Cardiomyocytederived DDT has autocrine effects activating stress signaling and limiting cardiac injury in vitro. (A) Generation of Myh6-Cre $D d t^{f / f l}$ mice. A Ddt targeting vector containing a 7-kb 5' arm and 3-kb 3' arm was generated to replace Ddt exons 1 and 2. 2 loxP sequences flanking exons 1 and 2 allowed for the creation of a cardiac-specific DDT knockout by intercross with Myh6-Cre recombinase mice. Deletion of exons 1 and 2 eliminated functional $D d t$ transcripts.

(B) DDT expression in $D d t^{f / f f}$, Myh6-Cre Ddt ${ }^{+/+}$, and Myh6-Cre $D d t^{f / f l}$ hearts was evaluated by immunohistochemistry with polyclonal anti-DDT antibody (nonimmune IgC antibody served as control). Arrow denotes remaining blood vessel DDT expression. Original magnification, $\times 10$. (C) Representative M-mode echocardiograms and corresponding values for $\mathrm{LV}$ dimensions and ejection fraction. (D-I) Ddt $t^{f / f l}$, Myh6-Cre Ddt t/+, and Myh6-Cre Ddt $t^{f / f l}$ hearts were subjected to ex vivo ischemia (15 minutes) and reperfusion (30 minutes). A separate group of Myh6-Cre Ddt $t^{f / f}$ hearts was also perfused with buffer containing rDDT (50 ng/ml). Cardiac contractile function was quantified as LVDP.HR index (D). Myocardial necrosis was determined by vital staining with TTC (E-H) and expressed as a percentage of ventricular tissue (I). Values are mean \pm SEM, $n=5-6$ per group. ${ }^{*} P<0.05$ vs. $D d t^{f / f l}$. action by incubating cardiomyocytes with the cell-permeant calcium chelator BAPTA-AM. We found that BAPTA-AM decreased AMPK activation after treatment with rDDT (Figure 4D), again implicating intracellular calcium as a component of the rDDT response (Figure $4 \mathrm{G}$ ).

Differential AMPK activation and cardiac injury after ischemia in $\mathrm{Mif}^{--}$and $\mathrm{Cd} 74^{-/-}$hearts. Recent findings indicate that anti-MIF antibodies fail to completely inhibit CD74-dependent cellular signaling responses and that $\mathrm{CD} 74$-deficient $\mathrm{B}$ cells are more sensitive to apoptosis than MIF-deficient cells $(28,29)$. We compared activation of the AMPK metabolic pathway during ischemia in hearts from WT, Mif ${ }^{--}$, and $C d 74^{-/}$mice. AMPK activation had a greater degree of impairment in $C d 74^{-/-}$versus $M i f^{--}$hearts during both in vitro and in vivo ischemia; as anticipated, both were reduced compared with WT hearts (Figure 5, A and C). LV contractile dysfunction was significantly more pronounced in $\mathrm{Cd} 74^{-/-}$compared with $\mathrm{Mif}^{/-}$isolated perfused hearts ( $36 \%$ vs. $58 \%$ recovery of LVDP.HR index) during reperfusion after ischemia (Figure $5 \mathrm{~B}$ and Supplemental Figure 7). Myocardial necrosis was also 2-fold more pronounced in $C d 74^{-/-}$mice than in $\mathrm{Mif}^{/-}$mice based on vital staining with TTC after coronary ligation and reperfusion (Figure 5D). These histological findings were paralleled by greater release of cardiac troponin I in serum of $\mathrm{Cd} 74^{-/-}$mice ( 10-fold increase over sham nonischemic controls) compared with $\mathrm{Mif}^{\prime-}$ mice ( 5-fold) (Figure 5E).

Together, these findings indicate that additional ligands trigger CD74 signaling responses in the heart, and we hypothesized 
A
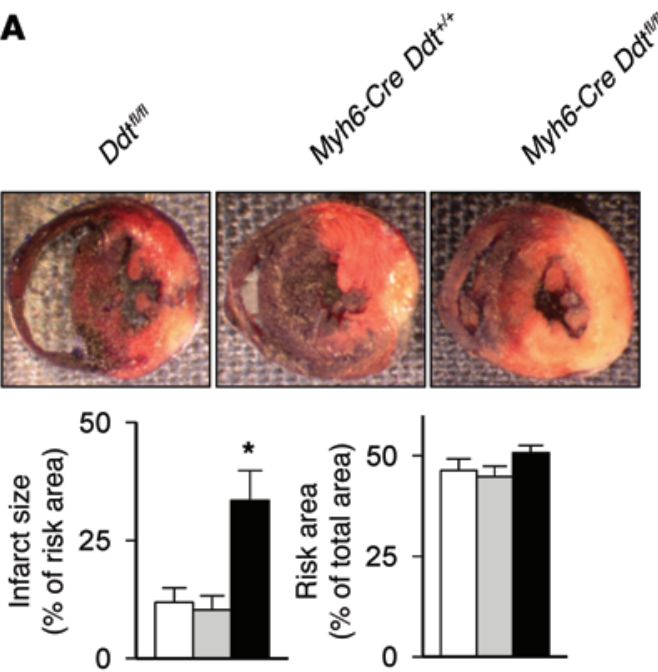

B

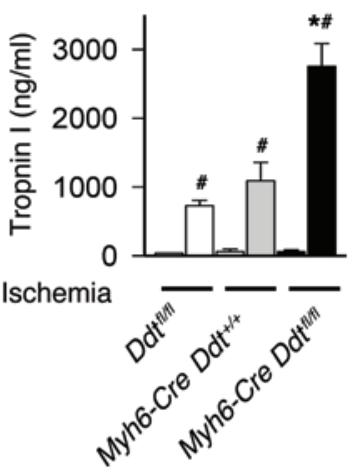

C

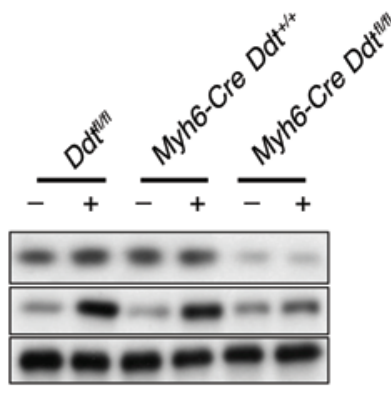

T-AMPK

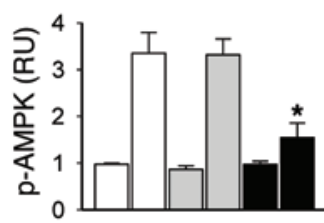

p-ACC

T-ACC

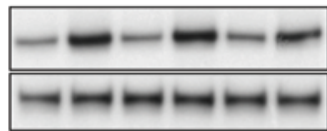

$D d t^{\text {HWT }}$

Myh6-Cre Ddt ${ }^{+/+}$

Myh6-Cre Ddt ${ }^{\text {tht }}$

D
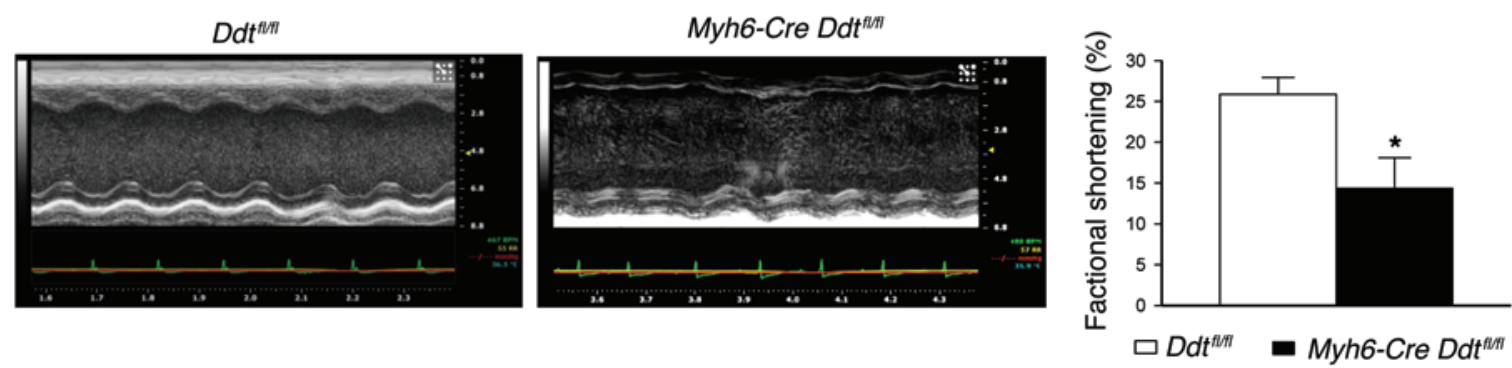

Figure 3. Cardiomyocyte-specific DDT deletion exacerbates cardiac injury after in vivo ischemia-reperfusion. Ddt ${ }^{f / f l}, \mathrm{Myh6}-\mathrm{Cre} D d t^{+/+}$, and $\mathrm{Myh6}-\mathrm{Cre}_{\mathrm{f}}$ $D d t^{f / f l}$ mice were subjected to left coronary artery ligation for 20 minutes with or without reperfusion, as indicated. (A) After 20 minutes of ischemia and 3 hours of reperfusion, myocardial necrosis (infarct size) was determined by TTC and Evans blue staining, and expressed as a percentage of the ischemic "risk area" served by the occluded artery. (B) Myocardial necrosis was also assessed by ELISA measurement of troponin I release into serum during reperfusion. (C) DDT expression and ischemic activation of AMPK and downstream ACC were assessed on immunoblots after 20 minutes of left coronary artery ligation without reperfusion. (D) Regional LV contractile function (fractional shortening) in vivo after 20 minutes of left coronary artery ligation and 24 hours of reperfusion, assessed by echocardiography. Data are mean \pm SEM, $n=5$ per group. ${ }^{*} P<0.05$ vs. Ddt ${ }^{f / / l}$ and $M y h 6-C r e D d t^{+/+}$; ${ }^{\#} P<0.05$ vs. nonischemic sham control.

that DDT might be a novel nonredundant ligand for CD74 in the heart. Thus, to directly test whether DDT signaling is mediated by $\mathrm{CD} 74$, we perfused isolated hearts with $\mathrm{rDDT}$ and found that AMPK activation was eliminated in $C d 74^{-/}$hearts (Figure $6 \mathrm{~A}$ and ref. 18). In separate control experiments, AMPK was similarly activated by metformin in WT and $C d 74^{-/}$hearts (data not shown), which indicates that $C d 74^{-/-}$hearts do not have a generalized defect in AMPK activation.

DDT treatment limits cardiac injury during in vitro ischemiareperfusion. We next hypothesized that pharmacologic treatment with rDDT might have beneficial protective effects in the ischemic heart. Initial experiments showed that administration of rDDT (at 10-fold greater concentrations than those required to activate AMPK) had no adverse effects on cardiac contractile function or heart rate (Figure 6A and Supplemental Figure 8A). DDT shares sequence homology to MIF, which is also expressed in cardiomyocytes and regulates AMPK and cellular metabo- lism (15) and limits ischemic injury (18). We compared the effects of rDDT with rMIF and found that both required CD74 for AMPK signaling (Figure 6, A and B). However, in contrast to rDDT, rMIF induced rapid LV contractile dysfunction (Figure 6B and Supplemental Figure 8B), as previously reported (19). Interestingly, experiments in $C d 74^{--}$mouse hearts demonstrated that the adverse contractile effects of MIF were CD74 independent (Figure 6B and Supplemental Figure 8B). DDT lacks the pseudo(E)LR motif present in MIF that mediates interaction with the chemokine receptors CXCR2 and CXCR4 (30), rendering DDT a more selective CD74 agonist than MIF. This selectivity could be highly advantageous in view of evidence that chemokine receptors exert pleiotropic effects in the heart, including a reduction in cardiac contractility and increased inflammation in the setting of ischemic injury $(31,32)$.

We then investigated whether pharmacologic treatment with rDDT protected the heart against ischemic injury after 
A
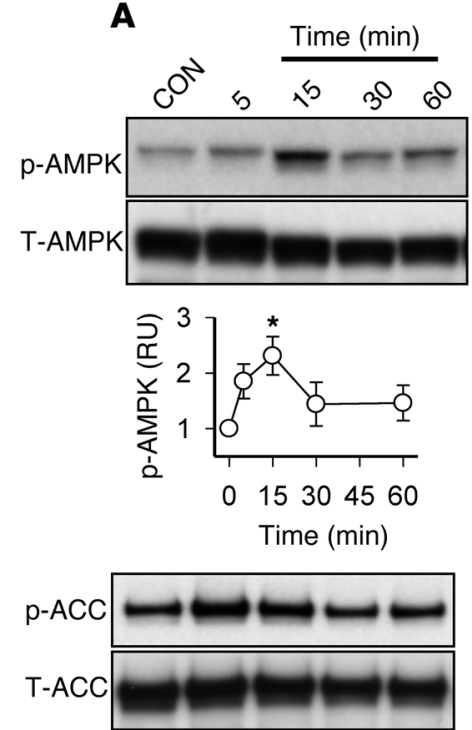

$\mathbf{E}$

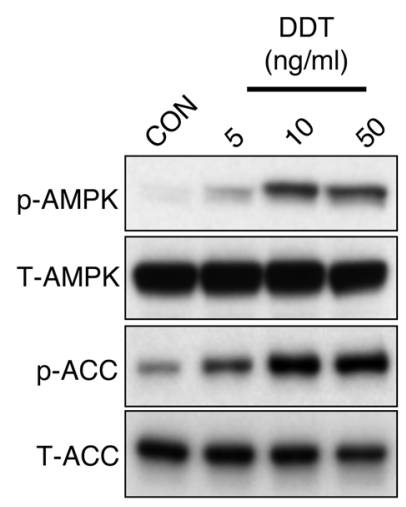

B
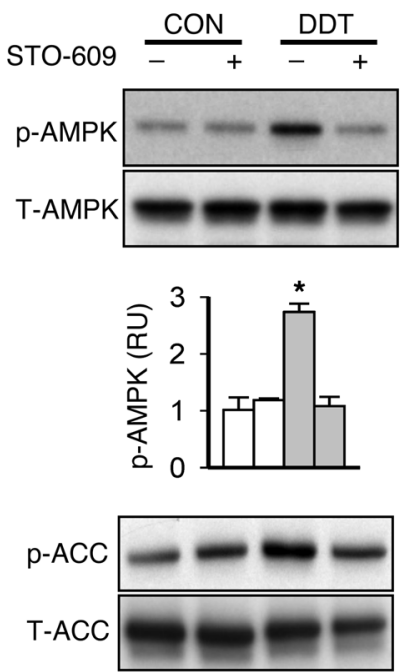

$\mathbf{F}$
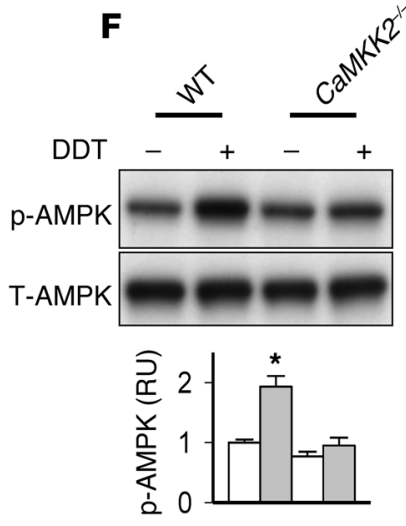

p-ACC

$\mathrm{T}-\mathrm{ACC}$
C
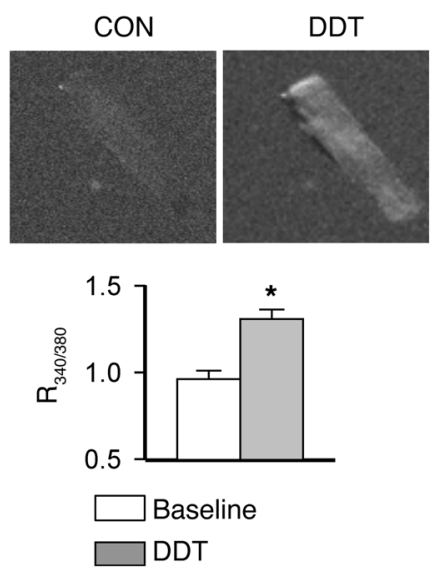
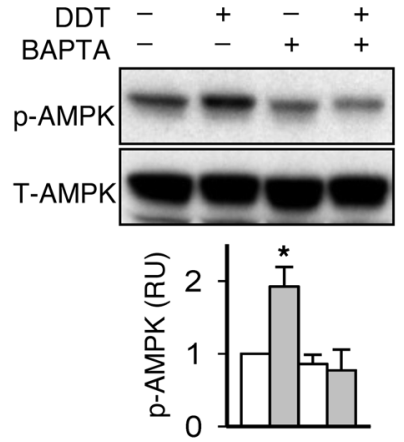

$\mathrm{p}-\mathrm{ACC}$

$\mathrm{T}-\mathrm{ACC}$

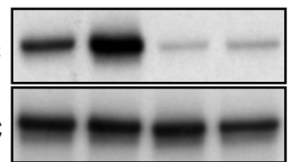

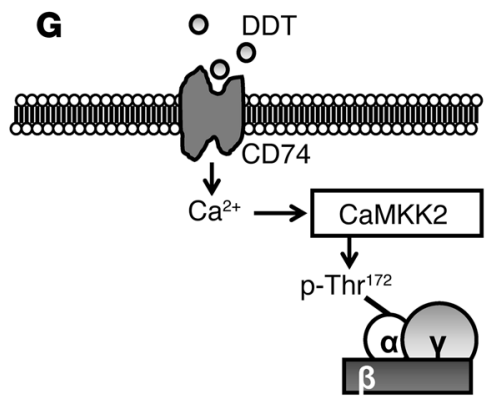

Figure 4. DDT activates AMPK through a CaMKK-dependent mechanism. (A) Time-dependent activation of the AMPK pathway in adult rat adult ventricular cardiomyocytes treated with recombinant DDT $(400 \mathrm{ng} / \mathrm{ml})$. (B) Effect of the CaMKK inhibitor STO-609 (2 $\mu$ M) on rDDT-induced AMPK activation in cardiomyocytes. (C) Intracellular calcium imaging was performed with fura-2-acetoxymethyl ester $(2 \mu \mathrm{M})$ in rat cardiomyocytes incubated without (baseline) or with rDDT $(400 \mathrm{ng} / \mathrm{ml})$. Original magnification, $\times 20$. The ratio of $340 \mathrm{~nm}$ to $380 \mathrm{~nm}$ fluorescence intensity $\left[\mathrm{R}_{(340 / 380)}\right.$ ] was recorded as a relative measure of intracellular calcium concentration. (D) Effect of binding intracellular calcium with BAPTA-AM $(10 \mu M)$ on rDDT stimulation of AMPK phosphorylation in cardiomyocytes. (E) WT mouse hearts were perfused with and without rDDT (5-50 ng/ml) for 15 minutes, and phospho- and total AMPK and ACC were assessed in immunoblots of heart homogenates. (F) DDT perfusion was also performed in Camkk2 $2^{-1-}$ hearts, and phospho- and total AMPK and ACC were subsequently assessed. (C) DDT activates AMPK through a $\mathrm{Ca}^{2+} / \mathrm{CaMKK}^{-}$dependent pathway. $\mathrm{p}^{- \text {Th }^{172}}{ }^{172}$, phosphorylation of AMPK at the Thr ${ }^{172}$ activating site in the catalytic domain. Data are mean \pm SEM, $n=4-6$ per group.

${ }^{*} P<0.05$ vs. respective control.

20 minutes of global ischemia and reperfusion. Direct treatment of isolated perfused hearts with $\mathrm{rDDT}(50 \mathrm{ng} / \mathrm{ml})$ enhanced the recovery of the LVDP.HR index by $65 \%$ as well as additional indices of cardiac contractile function (Figure 6C and Supplemental Figure 9). rDDT treatment also decreased myocardial necrosis by $70 \%$, as assessed by vital staining with TTC (Figure 6D). In order to determine the extent to which AMPK activation contributes to these cardioprotective actions of rDDT, we subjected AMPK kinase-dead mouse hearts (Tg $\left[C k m m-A m p k a^{K 45 R}\right]$; ref. 2) to ischemia-reperfusion. In contrast to WT hearts, rDDT did not protect against contractile dysfunction or necrosis in the AMPK- inactivated hearts after ischemia-reperfusion (Supplemental Figure 10, A and B), indicative of an important role for AMPK activation in DDT action.

\section{Discussion}

Our present results indicate that DDT is a novel secreted protein with important endogenous autocrine/paracrine actions during ischemia. There is no known physiologic substrate in mammalian species for DDT, and the protein appears to have evolved from a primitive enzymatic host defense pathway (16). Like MIF, DDT is an activating ligand for the mammalian CD74 receptor. DDT is 

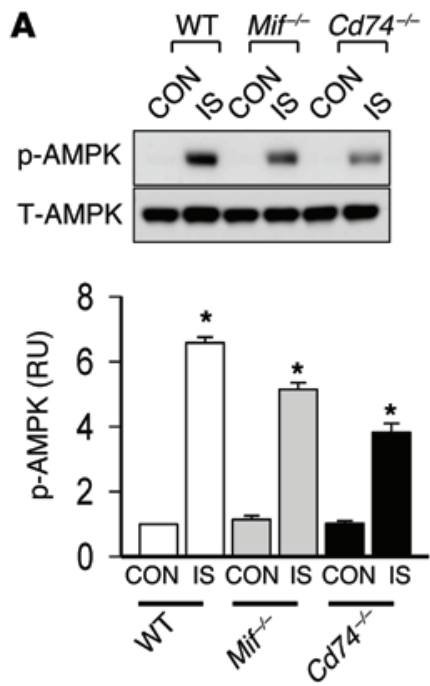

B

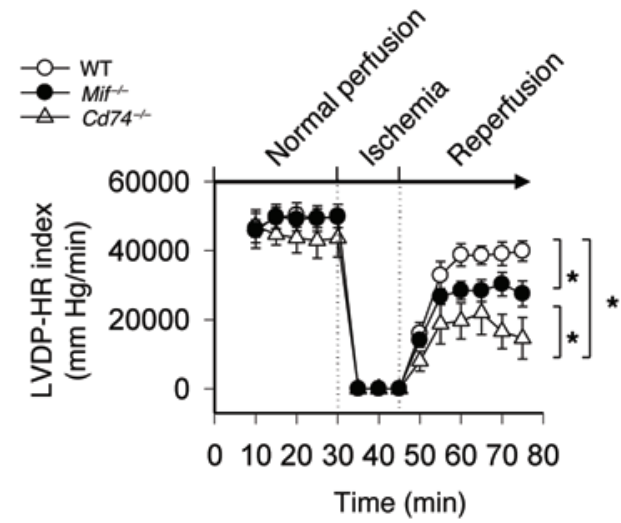

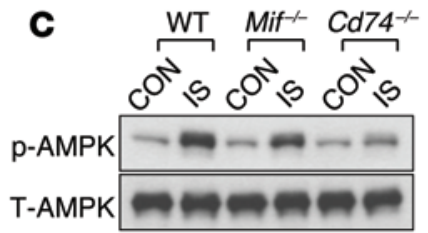

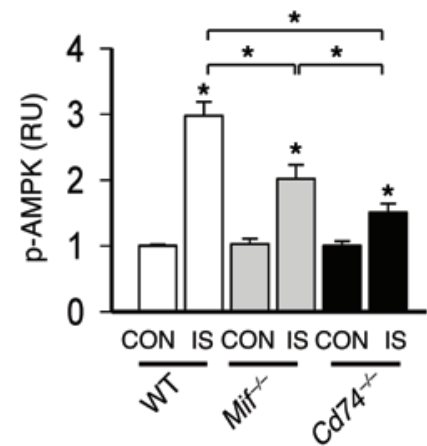

D

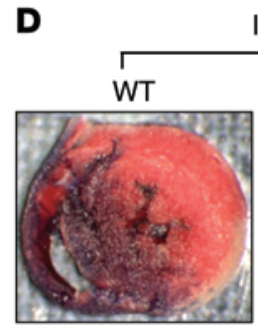

schemia-reperfusion
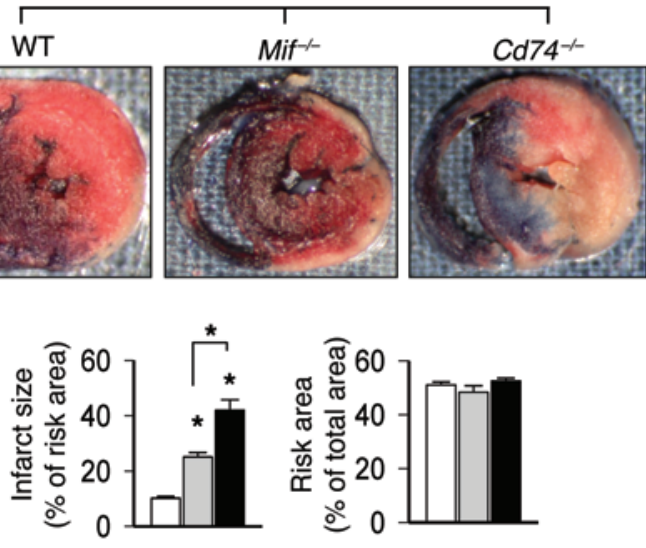

$\mathbf{E}$
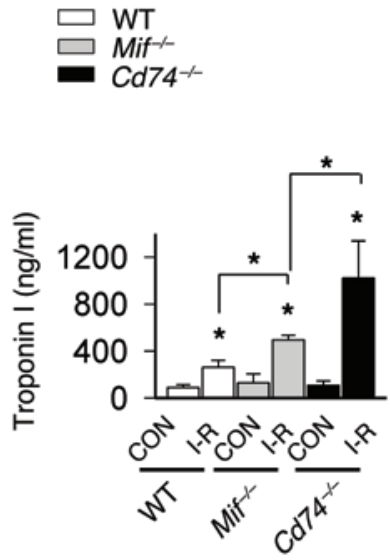

Figure 5. Differential AMPK activation and cardiac injury after ischemia in $\mathrm{Mif}^{-/}$and $\mathrm{Cd} 7 \mathbf{4}^{-/-}$hearts. (A) Hearts from WT, Mif ${ }^{-1}$, and $\mathrm{Cd} 74^{-/-}$mice were perfused ex vivo at normal flow for 30 minutes and then subjected to ischemia (15 minutes) with or without subsequent reperfusion (30 minutes). Control hearts were perfused at normal flow $(4 \mathrm{ml} / \mathrm{min})$ for 75 minutes. Heart homogenates were prepared after ischemia without reperfusion (IS) or control perfusion and immunoblotted with phospho-AMPK (Thr ${ }^{172}$ ) and total AMPK antibodies. (B) LVDP•HR index during the in vitro perfusion protocols. (C) Mice underwent ligation of the left coronary artery (or control sham ligation) for 20 minutes with or without subsequent reperfusion for 3 hours. PhosphoAMPK (Thr ${ }^{172}$ ) and total AMPK were assessed in heart homogenates after ischemia without reperfusion. (D) Myocardial necrosis, determined by TTC and Evans blue staining and expressed as a percentage of the ischemic "risk area" served by the occluded artery. (E) Myocardial necrosis was also assessed by measurement of serum troponin I after ischemia-reperfusion (I-R) or sham surgery. Data are mean $\pm \mathrm{SEM}, n=5-6$ per group. ${ }^{*} P<0.05$ vs. respective control or as indicated by brackets.

secreted from cardiomyocytes and has a physiologically important role in modulating metabolic stress signaling and protecting against ischemic necrosis. Our findings define a DDT/CD74/ CaMKK2/AMPK pathway in the heart, for the first time elucidating a signal transduction mechanism between autocrine/paracrine factors and AMPK activation. In addition, our results provide initial proof of concept that pharmacologic treatment with rDDT protects the heart against injury during ischemia-reperfusion. The characteristics of selective CD74 specificity and lack of adverse effects on cardiac physiologic function make DDT a potentially attractive treatment strategy to prevent solid organ ischemic injury.

Endogenous secreted DDT modulated AMPK activation during ischemia-reperfusion, based on both our neutralizing antibody studies and experiments in mouse hearts with cardiomyocyte DDT deletion. AMPK is a stress kinase known to play a key role in regulating cardiac metabolism and preventing ischemic heart damage (2). The intrinsic AMPK pathway is activated and facilitates glucose metabolism during ischemia, and limits apoptosis and necrosis during ischemia-reperfusion (2). We also successfully generated endotoxin-free, biologically active rDDT, which protected perfused hearts against contractile dysfunction and myocardial necrosis during ischemia-reperfusion. This cardioprotective effect appeared to require downstream AMPK activation, based on its lack of efficacy in mice with genetic inactivation of AMPK in their hearts.

Our studies also dissected out the mechanisms linking DDT and AMPK activation in the heart. AMPK is a heterotrimer with 

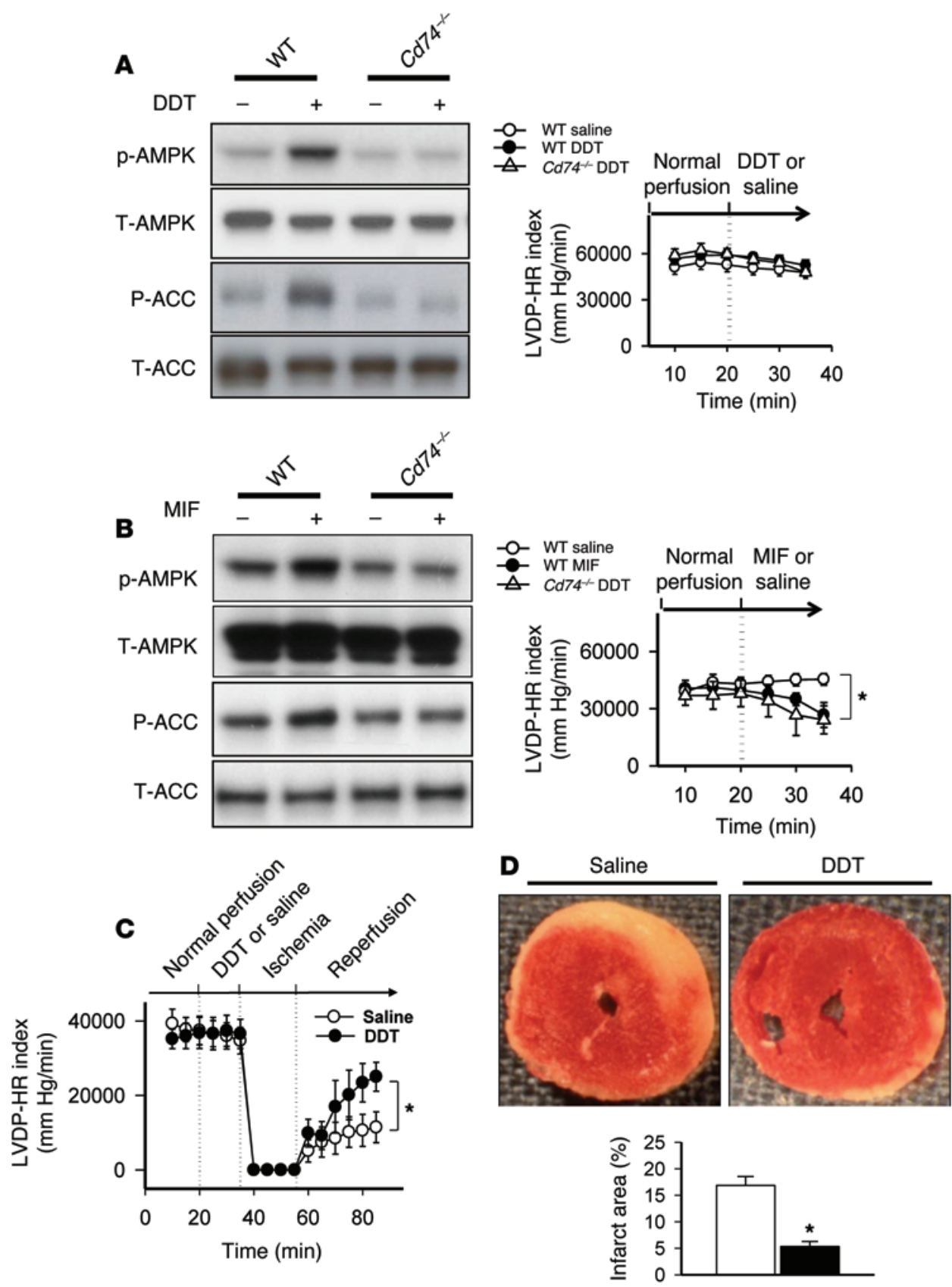

Figure 6. rDDT treatment activates AMPK through CD74 and limits cardiac injury during ischemia-reperfusion.

(A and B) WT and $\mathrm{Cd} 74^{-/-}$mouse hearts were perfused with saline (control) or with $50 \mathrm{ng} / \mathrm{ml} \mathrm{rDDT} \mathrm{(A)} \mathrm{or} \mathrm{rMIF} \mathrm{(B)} \mathrm{for}$ 15 minutes. Phospho- and total AMPK and ACC were assessed in immunoblots of heart homogenates, and cardiac contractile function was evaluated (LVDP•HR index). (C) Hearts isolated from WT mice were treated with saline or $50 \mathrm{ng} / \mathrm{ml}$ rDDT for 15 minutes and then subjected to 20 minutes of global ischemia followed by 30 minutes of reperfusion. Cardiac contractile function was evaluated (LVDP•HR index). (D) In these hearts, TTC staining was used to quantify the extent of necrosis (infarct area calculated as a percentage of ventricular area). Data are mean \pm SEM, $n=5-6$ per group. ${ }^{*} P<0.05$ vs. control. an $\alpha$ catalytic subunit and regulatory $\beta$ and $\gamma$ subunits (1). AMPK is activated by LKB1 and CaMKK2, which phosphorylate the $\mathrm{Thr}^{172}$ activating site in the $\alpha$ subunit of AMPK (1). Our experiments implicate CaMKK2 as the primary upstream kinase responsible for DDT activation of AMPK (Figure $4 \mathrm{G}$ ). $\mathrm{Ca}^{2+} /$ calmodulin binding to CaMKK2 is necessary for AMPK activation (33) in a multiprotein complex that includes $\mathrm{Ca}^{2+} /$ calmodulin, CaMKK2, and AMPK subunits (27). CaMKK2-mediated AMPK activation is AMP independent, in contrast to LKB1 activation of AMPK, which requires AMP or ADP interaction with the nucleotide-binding domains of the $\gamma$ subunit (27). Our findings suggest that DDT might mediate a relatively slow, but brief (1-2 minutes), increase in intracellular diastolic calcium, and also set the stage for additional studies to define the subcellular localization of CaMKK and the source of calcium responsible for CaMKK/AMPK activation in the heart. Thus, we postulate that the amplification of AMPK activity by DDT, and potentially other autocrine/paracrine effects, may occur via a calcium-dependent, energy-independent mechanism.

The protective effect of the DDT/CD74/CaMKK2/AMPK pathway that we observed in the ischemic heart is of interest, in view of recent findings that CaMKK2 and downstream CaMKIV limit hepatic damage during ischemia-reperfusion (34). These protective actions of CaMKK2 are distinct from the effects of other $\mathrm{Ca}^{2+} /$ calmodulin-dependent kinases during ischemia. In particular, they contrast with the detrimental effects of the CaMKK2independent CaMKII pathway, which is activated by generalized calcium overload and increases cytotoxic mitochondrial calcium overload during ischemia-reperfusion (35). Inhibition of CaMKII 
has been proposed as a strategy for preventing ischemic injury and arrhythmogenesis (35).

DDT shares limited sequence homology with MIF, and recent studies suggest the possibility that DDT might also have CD74dependent effects on macrophages (22), as well as metabolic effects in adipocytes (36) and cancer cells (37). In obesity, DDT expression is downregulated in adipose tissue, where it has an antilipolytic effect to inhibit hormone-sensitive lipase (36). This action of DDT was thought to be due to the combined inhibition of protein kinase A and activation of AMPK, although DDT administration to $d b / d b$ mice did not itself promote AMPK Thr ${ }^{172}$ phosphorylation (36). Interestingly, in rapidly dividing A549 lung adenocarcinoma cells, CD74 activation by either MIF or DDT paradoxically decreased AMPK activation, reportedly by increasing glucose uptake and ATP production and inhibiting CaMKK2 activation (37). Whether these effects of CD74 activation reflect specific tissue-related or cell transformation-related differences remains to be elucidated.

The finding that DDT acts as a second endogenous ligand for CD74 in the heart accounts for the increased susceptibility to cardiac ischemic injury that we observed in $\mathrm{Cd} 74^{-/}$compared with Mif/- mouse hearts. MIF is a cognate ligand for CD74 that activates AMPK in the heart (15) and liver (38). Although MIF and DDT each requires CD74 to activate AMPK, these mediators do not have simply redundant actions, but exhibit important differences in their physiologic actions. Specifically, DDT does not share the negative inotropic effect of MIF to decrease cardiac contractility $(18,19)$. The ability of both MIF and DDT to activate AMPK is most likely mediated by structurally homologous $\mathrm{N}$-terminal domains $(39,40)$, while the decrease in contractility seen with MIF may be due to its additional interaction with the noncognate chemokine receptors CXCR2 and CXCR4 (30). MIF interacts with these latter receptors through a pseudo(E)LR domain, which is not present in DDT (22). DDT also exhibits distinct CD74-binding kinetics compared with MIF, which may account for its more rapid activation of AMPK (22). In vivo, both the kinetics of CD74 signaling and the downstream response of the heart will likely reflect the interplay between MIF and DDT concentrations in the interstitial space. Nevertheless, the distinctive features of DDT signaling first described herein - selective receptor activation and more rapid AMPK activation kinetics are advantageous characteristics in the consideration of potential cardioprotective pharmacologic strategies.

Our studies provide initial evidence that rDDT treatment has a cardioprotective effect during ischemia-reperfusion in normal mouse hearts. Further studies will be required to address whether rDDT is effective in diabetes, aging, and other pathologic conditions that can potentially decrease the efficacy of cardioprotective strategies. Additional investigation will also be required to compare rDDT with other interventions that activate AMPK, including metformin (41) and other metabolic therapies, as well as to determine its efficacy in combination with these therapies. It is worth noting that glucose-insulin-potassium (GIK) was successful in preventing ischemic injury in animal studies $(42,43)$, but did not prove efficacious in large clinical trials (44). Although DDT and insulin both activate heart glucose uptake, DDT action is mediated via AMPK, and insulin action by PI3K (1). The actions of these signaling pathways fundamentally differ in many other respects: AMPK conserves ATP by promoting substrate catabolism, inhibiting protein synthesis, and stimulating autophagy, whereas PI3K stimulates glucose and fatty acid storage, stimulates protein synthesis, and inhibits autophagy through the activation of mTOR (1).

Thus, our present findings demonstrate that DDT is a novel secreted cardiac protein with important autocrine/paracrine effects during ischemia and cardioprotective effects when administered as a recombinant protein during ischemia-reperfusion. Additional research is required to further determine the potential for developing DDT as a pharmacologic strategy for protecting the heart and other solid organs against ischemic injury.

\section{Methods}

Experimental animals. Animals were fed and housed according to the guidelines of the American Association for Laboratory Animal Care. WT BALB/c mice (age 10-16 weeks) and Sprague-Dawley rats (220-250 g) were obtained from Charles River Laboratory. Mif ${ }^{\prime-}$ and $C d 74^{-/-}$mice were described previously $(15,45)$. AMPK kinase-dead (Tg[Ckmm-Ampka $\left.{ }^{K 45 R}\right]$; ref. 2) and Camkk2-/- (gift of A.R. Means, Duke University School of Medicine, Durham, North Carolina, USA; ref. 27) mice were fully backcrossed into a C57BL/6 background.

Generation of Myh6-Cre Ddt/f/t mice. Genomic DNA fragments containing the Ddt gene were isolated from C57BL/6 BAC clone RP2350I3, RP23-412I6, and RP23-55M4. The final targeting vectors were flanked with $5^{\prime}$ and 3 ' arms and contained genes for puromycin resistance and tyrosine kinase for positive and negative selection of targeted ES cell clones. A $D d t$ targeting vector was designed to replace $D d t$ exons 1 and 2 and contained 2 loxP sequences flanking exons 1 and 2; these are recognized by Cre recombinase for conditional gene deletion of the intervening exons (Figure 2A).

The targeting vectors were transfected into mouse V6.5 ES cells (male C57BL/6 $\times$ female 129/SV) (Taconic Artemis). A positive and negative selection strategy with puromycin and ganciclovir was used to eliminate untargeted and nonhomologous recombinant ES cells. The homologous recombinants were selected by Southern blot analysis. Targeted ES clones were verified by sequencing and microinjected into BALB/c blastocysts. Chimeric mice with germline transmission were identified, and offspring were bred to homozygosity to produce a population of $D d t^{f / f l}$ mice in the pure C57BL/6 background. In order to obtain the cardiac-specific DDT knockout, $D d t^{f / f}$ mice were crossed with Myh6-Cre mice, and the Myh6-Cre $D d t^{f /+}$ offspring were then crossed to obtain homozygous Ddt t/f/, Myh6-Cre Ddt $t^{A / f}$, and Myh6-Cre D $d t^{+/+}$mice.

$M I F$ and DDT. Mouse rMIF and rDDT were produced and purified as previously described $(22,46)$.

Antibodies. Neutralizing anti-MIF monoclonal (IgG1) and anti-DDT polyclonal (IgG fraction) antibodies were purified from mouse ascites and rabbit immune serum, respectively (22). Primary antibodies used for immunoblotting were directed against AMPK $\alpha$ subunit phosphorylated at the Thr ${ }^{172}$ site, total AMPK $\alpha$ subunit, ACC phosphorylated at the Ser ${ }^{79}$ site, and total ACC (Cell Signaling). Secondary antibody was HRP-linked anti-rabbit or anti-mouse IgG (Cell Signaling).

Isolated heart perfusions. Mice were heparinized (100 U i.p.) and anesthetized with pentobarbital sodium (60 mg/kg i.p.), and the heart was quickly excised and perfused in the Langendorff mode with oxygenated Krebs-Henseleit buffer containing $7 \mathrm{mmol} / \mathrm{l}$ glucose, $0.4 \mathrm{mmol} / \mathrm{l}$ oleate, $1 \% \mathrm{BSA}$, and $10 \mu \mathrm{U} / \mathrm{ml}$ insulin (15). A 
balloon was placed in the LV and inflated to a diastolic pressure of $5 \mathrm{~mm} \mathrm{Hg}$ during baseline perfusion, and LVDP and HR were monitored (AD heart perfusion instruments) (15). For ischemia-reperfusion studies, hearts were stabilized at a flow rate of $4 \mathrm{ml} / \mathrm{min}$ for 30 minutes prior to 15 minutes of no-flow global ischemia, followed by 30 minutes of reperfusion. The extent of myocardial necrosis was evaluated by TTC staining of viable tissue at the end of the experiment (15). For rDDT or rMIF treatment studies, perfused hearts were stabilized for 20 minutes, then subjected to treatment as specified in the figure legends.

Coronary ligation and reperfusion in vivo. Mice were anesthetized with pentobarbital sodium (60 mg/kg i.p.), intubated, and ventilated with room air supplemented with oxygen (18). After surgical thoracotomy, the left coronary artery was ligated $2 \mathrm{~mm}$ below the left atrial appendage for 20 minutes and then reperfused for 3 hours (18). In sham operations, sutures were placed without ligation. The core temperature of the mice was maintained throughout the protocol. An electrocardiogram was monitored to confirm ischemic ST segment elevation during coronary artery occlusion. Myocardial necrosis was evaluated by TTC staining in the ischemic area perfused by the ligated coronary; the nonischemic region was delineated by infusion of Evans blue stain (18). Serum troponin was also measured during reperfusion as an index of myocardial necrosis (18).

Echocardiography. Mice were anesthetized with 1\%-2\% (v/v) isoflurane and then underwent 2D and M-mode echocardiography (VisualSonics 2100), maintaining core temperature with heating blankets, as previously described (47). Baseline LV structure (dimension and wall thickness) and function (fractional shortening) were assessed in $D d t^{f / f}$, Myh6-Cre Ddt $t^{f / f}$, and Myh6-Cre Ddt $t^{+/+}$mice at 10-12 weeks of age. Cardiac LV contractile function was also assessed in $D d t^{t / / f}$ and Myh6-Cre Ddt/f/l mice after 20 minutes of coronary occlusion and 24 hours of reperfusion using echocardiography (VisualSonics 2100), as previously described (48). LV fractional shortening at the midventricular and apical levels was assessed from a long-axis view with anatomic M-mode imaging.

Immunohistochemistry. Hearts were fixed by formalin perfusion, embedded with paraffin, and sliced into $3-\mu \mathrm{m}$ sections (15). Sections were incubated with primary anti-DDT or anti-MIF antibodies overnight (or nonspecific IgG antibody for control), treated with secondary antibody, and visualized with the Liquid DAB + Substrate Chromogen System (Dako Cytomation).
Cardiomyocyte isolation. Adult ventricular cardiomyocytes were isolated from rats using collagenase-containing buffer (49). Cardiomyocytes were incubated in $95 \%$ room air and $5 \% \mathrm{CO}_{2}$ at $37^{\circ} \mathrm{C}$ for approximately 3 hours, and the medium was changed to remove nonattached dead cells.

Calcium imaging. Isolated adult rat cardiomyocytes were loaded with $2 \mu \mathrm{M}$ fura-2-acetoxymethyl ester (Invitrogen) dissolved in HEPES buffer (containing $145 \mathrm{mM} \mathrm{NaCl}, 3 \mathrm{mM} \mathrm{KCl}, 2 \mathrm{mM} \mathrm{MgCl}_{2}, 2 \mathrm{mM} \mathrm{CaCl}_{2}$, $10 \mathrm{mM}$ glucose, and $10 \mathrm{mM}$ HEPES; adjusted to $\mathrm{pH} 7.4$ with $\mathrm{NaOH}$ ) in the darkfor 45 minutes at $37^{\circ} \mathrm{C}$, and cells were excited at 340 and $380 \mathrm{~nm}$ using a Polychrome V Monochromator (TILL Photonics). Images were recorded at 2-second intervals at room temperature $\left(20^{\circ} \mathrm{C}-22^{\circ} \mathrm{C}\right)$ using a cooled CCD camera (Sensicam) controlled by a computer with Image Workbench 5.2 software (Indec Biosystems). The ratio of $340 \mathrm{~nm}$ to $380 \mathrm{~nm}$ fluorescence intensity was recorded as a relative measure of intracellular calcium concentration (50).

Measurement of DDT and MIF. Concentrations of DDT and MIF in coronary venous effluents were measured by specific ELISA assays, as previously described (22).

Measurement of heart muscle glucose uptake. Glucose uptake in rat papillary muscle was measured as described previously (2).

Statistics. All values are mean \pm SEM. GraphPad Prism software was used for statistical analysis. ANOVA with subsequent Tukey test was used to determine differences among 3 or more groups and for comparison of multiple time points. 2-tailed Student's $t$ test was used for comparison of 2 groups. A $P$ value less than 0.05 was considered statistically significant.

Study approval. All animal procedures were approved by the Yale University Animal Care and Use Committee.

\section{Acknowledgments}

We appreciate the contributions from Yu Jin, Alda Bregasi, and Kevin N. Su. The studies described herein were supported in part by NIH grants R01 HL-63811 and AI-042310 and by American Heart Association grant 13GRNT17090068.

Address correspondence to: Lawrence H. Young, Yale Cardiovascular Research Center, 300 George Street, New Haven, Connecticut 06519, USA. Phone: 203.785.4102; E-mail: lawrence.young@yale.edu.
1. Zaha VG, Young LH. AMP-activated protein kinase regulation and biological actions in the heart. Circ Res. 2012;111(6):800-814.

2. Russell RR, et al. AMP-activated protein kinase mediates ischemic glucose uptake and prevents postischemic cardiac dysfunction, apoptosis, and injury. J Clin Invest. 2004;114(4):495-503.

3. Russell RR, Russell RR 3rd, Bergeron R, Shulman GI, Young LH. Translocation of myocardial GLUT-4 and increased glucose uptake through activation of AMPK by AICAR. Am J Physiol. 1999;277(2 pt 2):H643-H649.

4. Marsin AS, Bouzin C, Bertrand L, Hue L. The stimulation of glycolysis by hypoxia in activated monocytes is mediated by AMP-activated protein kinase and inducible 6-phosphofructo-2-kinase. J Biol Chem. 2002;277(34):30778-30783.

5. Horman S, et al. Activation of AMP-activated protein kinase leads to the phosphorylation of elongation factor 2 and an inhibition of protein synthesis. Curr Biol. 2002;12(16):1419-1423.

6. Muoio DM, Seefeld K, Witters LA, Coleman RA. AMP-activated kinase reciprocally regulates triacylglycerol synthesis and fatty acid oxidation in liver and muscle: evidence that sn-glycerol3-phosphate acyltransferase is a novel target. Biochem J. 1999;338(pt 3):783-791.

7. Matsui Y, et al. Distinct roles of autophagy in the heart during ischemia and reperfusion: roles of AMP-activated protein kinase and Beclin 1 in mediating autophagy. Circ Res. 2007; 100(6):914-922.

8. Wang Y, et al. AMP-activated protein kinase deficiency enhances myocardial ischemia/reperfusion injury but has minimal effect on the antioxidant/antinitrative protection of adiponectin.
Circulation. 2009;119(6):835-844.

9. Paiva MA, et al. Enhancing AMPK activation during ischemia protects the diabetic heart against reperfusion injury. Am J Physiol Heart Circ Physiol. 2011;300(6):H2123-H2134.

10. Carvajal K, et al. Dual cardiac contractile effects of the alpha2-AMPK deletion in low-flow ischemia and reperfusion. Am J Physiol Heart Circ Physiol. 2007;292(6):H3136-H3147.

11. Minokoshi Y, et al. Leptin stimulates fatty-acid oxidation by activating AMP-activated protein kinase. Nature. 2002;415(6869):339-343.

12. Yamauchi T, et al. Adiponectin stimulates glucose utilization and fatty-acid oxidation by activating AMP-activated protein kinase. Nat Med. 2002;8(11):1288-1295.

13. Tao L, et al. Adiponectin cardioprotection after myocardial ischemia/reperfusion involves the 
reduction of oxidative/nitrative stress. Circulation. 2007;115(11):1408-1416.

14. Oshima Y, Ouchi N, Sato K, Izumiya Y, Pimentel DR, Walsh K. Follistatin-like 1 is an Akt-regulated cardioprotective factor that is secreted by the heart. Circulation. 2008;117(24):3099-3108.

15. Miller EJ, et al. Macrophage migration inhibitory factor stimulates AMP-activated protein kinase in the ischaemic heart. Nature. 2008;451(7178):578-582.

16. Merk M, Mitchell RA, Endres S, Bucala R. D-dopachrome tautomerase (D-DT or MIF-2): doubling the MIF cytokine family. Cytokine. 2012;59(1):10-17.

17. Gao XM, et al. Deletion of macrophage migration inhibitory factor protects the heart from severe ischemia-reperfusion injury: a predominant role of anti-inflammation. J Mol Cell Cardiol. 2011;50(6):991-999.

18. Qi D, et al. Cardiac macrophage migration inhibitory factor inhibits JNK pathway activation and injury during ischemia/reperfusion. JClin Invest. 2009;119(12):3807-3816.

19. Garner LB, et al. Macrophage migration inhibitory factor is a cardiac-derived myocardial depressant factor. Am J Physiol Heart Circ Physiol. 2003;285(6):H2500-H2509.

20. Odh G, Hindemith A, Rosengren AM, Rosengren $\mathrm{E}$, Rorsman $\mathrm{H}$. Isolation of a new tautomerase monitored by the conversion of D-dopachrome to 5,6-dihydroxyindole. Biochem Biophys Res Commun. 1993;197(2):619-624.

21. Sugimoto H, Taniguchi M, Nakagawa A, Tanaka I, Suzuki M, Nishihira J. Crystal structure of human D-dopachrome tautomerase, a homologue of macrophage migration inhibitory factor, at $1.54 \mathrm{~A}$ resolution. Biochemistry. 1999;38(11):3268-3279.

22. Merk M, et al. The D-dopachrome tautomerase (DDT) gene product is a cytokine and functional homolog of macrophage migration inhibitory factor (MIF). Proc Natl Acad Sci U S A. 2011;108(34):E577-E585.

23. Woods A, et al. Ca2+/calmodulin-dependent protein kinase kinase-beta acts upstream of AMPactivated protein kinase in mammalian cells. Cell Metab. 2005;2(1):21-33.

24. Hawley SA, et al. Calmodulin-dependent protein kinase kinase-beta is an alternative upstream kinase for AMP-activated protein kinase. Cell Metab. 2005;2(1):9-19.

25. Tokumitsu H, Inuzuka H, Ishikawa Y, Ikeda M, Saji I, Kobayashi R. STO-609, a specific inhibitor of the $\mathrm{Ca}(2+) /$ calmodulin-dependent protein kinase kinase. J Biol Chem. 2002;277(18):15813-15818.
26. Bain J, et al. The selectivity of protein kinase inhibitors: a further update. Biochem J. 2007;408(3):297-315

27. Anderson KA, et al. Hypothalamic CaMKK2 contributes to the regulation of energy balance. Cell Metab. 2008;7(5):377-388.

28. Chagnon F, Metz CN, Bucala R, Lesur O. Endotoxin-induced myocardial dysfunction: effects of macrophage migration inhibitory factor neutralization. Circ Res. 2005;96(10):1095-1102.

29. Gore Y, et al. Macrophage migration inhibitory factor induces B cell survival by activation of a CD74-CD44 receptor complex. J Biol Chem. 2008;283(5):2784-2792.

30. Weber C, et al. Structural determinants of MIF functions in CXCR2-mediated inflammatory and atherogenic leukocyte recruitment. Proc Natl Acad Sci U S A. 2008;105(42):16278-16283.

31. Tarzami ST, et al. Opposing effects mediated by the chemokine receptor CXCR2 on myocardial ischemia-reperfusion injury: recruitment of potentially damaging neutrophils and direct myocardial protection. Circulation. 2003;108(19):2387-2392.

32. Larocca TJ, et al. CXCR4 gene transfer prevents pressure overload induced heart failure. J Mol Cell Cardiol. 2012;53(2):223-232.

33. Racioppi L, Means AR. Calcium/calmodulindependent protein kinase kinase 2: roles in signaling and pathophysiology. J Biol Chem. 2012;287(38):31658-31665.

34. Evankovich J, et al. Calcium/calmodulin-dependent protein kinase IV limits organ damage in hepatic ischemia-reperfusion injury through induction of autophagy. Am J Physiol Gastrointest Liver Physiol. 2012;303(2):G189-G198.

35. Joiner ML, et al. CaMKII determines mitochondrial stress responses in heart. Nature. 2012;491(7423):269-273.

36. Iwata $\mathrm{T}$, et al. The action of $\mathrm{D}$-dopachrome tautomerase as an adipokine in adipocyte lipid metabolism. PLoS One. 2012;7(3):e33402.

37. Brock SE, Rendon BE, Yaddanapudi K, Mitchell RA. Negative regulation of AMP-activated protein kinase (AMPK) activity by macrophage migration inhibitory factor (MIF) family members in non-small cell lung carcinomas. J Biol Chem. 2012;287(45):37917-37925.

38. Heinrichs D, et al. Macrophage migration inhibitory factor (MIF) exerts antifibrotic effects in experimental liver fibrosis via CD74. Proc Natl Acad Sci U S A. 2011;108(42):17444-17449.

39. Bendrat K, et al. Biochemical and mutational investigations of the enzymatic activity of macro- phage migration inhibitory factor. Biochemistry. 1997;36(49):15356-15362.

40. Fingerle-Rowson G, et al. A tautomerase-null macrophage migration-inhibitory factor (MIF) gene knock-in mouse model reveals that protein interactions and not enzymatic activity mediate MIF-dependent growth regulation. Mol Cell Biol. 2009;29(7):1922-1932.

41. Calvert JW, et al. Acute metformin therapy confers cardioprotection against myocardial infarction via AMPK-eNOS-mediated signaling. Diabetes. 2008;57(3):696-705.

42. Zhang HX, et al. Physiologically tolerable insulin reduces myocardial injury and improves cardiac functional recovery in myocardial ischemic/ reperfused dogs. J Cardiovasc Pharmacol. 2006;48(6):306-313.

43. Hafstad AD, Khalid AM, How OJ, Larsen TS, Aasum E. Glucose and insulin improve cardiac efficiency and postischemic functional recovery in perfused hearts from type 2 diabetic (db/db) mice. Am J Physiol Endocrinol Metab. 2007;292(5):E1288-E1294.

44. Selker HP, et al. Out-of-hospital administration of intravenous glucose-insulin-potassium in patients with suspected acute coronary syndromes: the IMMEDIATE randomized controlled trial. JAMA. 2012;307(18):1925-1933.

45. Shi X, et al. CD44 is the signaling component of the macrophage migration inhibitory factor-CD74 receptor complex. Immunity. 2006;25(4):595-606.

46. Bernhagen J, Mitchell RA, Calandra T, Voelter W, Cerami A, Bucala R. Purification, bioactivity, and secondary structure analysis of mouse and human macrophage migration inhibitory factor (MIF). Biochemistry. 1994; 33(47):14144-14155.

47. Hedhli N, et al. Endothelium-derived neuregulin protects the heart against ischemic injury. Circulation. 2011;123(20):2254-2262.

48. Li J, et al. Urocortin 2 autocrine/paracrine and pharmacologic effects to activate AMP-activated protein kinase in the heart. Proc Natl Acad SciU S A. 2013;110(40):16133-16138.

49. An D, et al. Metformin influences cardiomyocyte cell death by pathways that are dependent and independent of caspase-3. Diabetologia. 2006;49(9):2174-2184.

50. Qu L, Li Y, Pan X, Zhang P, LaMotte RH, Ma C. Transient receptor potential canonical 3 (TRPC3) is required for IgG immune complex-induced excitation of the rat dorsal root ganglion neurons. JNeurosci. 2012;32(28):9554-9562. 